\title{
Biochemical and virological analysis of the preference for the K65R multi-resistance nucleoside mutation in subtype $\mathbf{C}$ viruses Mark A Wainberg*, Cédric F Invernizzi, Dimitrios Coutsinos, Maureen Oliveira, Daniela Moisi and Bluma G Brenner
}

Address: McGill University AIDS Centre, Jewish General Hospital, Montréal, Québec, Canada

* Corresponding author

from Frontiers of Retrovirology: Complex retroviruses, retroelements and their hosts

Montpellier, France. 2I-23 September 2009

Published: 24 September 2009

Retrovirology 2009, 6(Suppl 2):II8 doi:10.1 |86/I742-4690-6-S2-II8

This abstract is available from: http://www.retrovirology.com/content/6/S2/II8

(C) 2009 Wainberg et al; licensee BioMed Central Ltd.

\section{Objectives}

Patients carrying subtype $\mathrm{C}$ viruses in Botswana, Malawi, and South Africa are prone to develop the K65R mutation in $\mathrm{RT}$ after receiving $\mathrm{d} 4 \mathrm{~T}$ or $\mathrm{ddI} / \mathrm{d} 4 \mathrm{~T}$ in therapy. We have studied the effect of subtype $C$ polymorphisms at position $64 / 65$ and at TAM sites $(70,210,219)$ in RT on pathway outcome in cell culture, utilizing $\mathrm{N}(\mathrm{t}) \mathrm{RTI}$ drug pressure alone and in combination.

\section{Methods}

We compared mutations of the subtype B NL4-3 (wt) sequence at sites 64, 65, 70, 210, 219 with silent polymorphisms found in the subtype $\mathrm{C}$ consensus sequence. Sitedirected mutagenesis of the NL4-3 (wt) plasmid was used to generate all mutant plasmids. Selections in MT-2 cells were performed with each of 3TC, FTC, TDF, ABC, ATC, $\mathrm{d} 4 \mathrm{~T}$, and ddI either alone or in combination. Recombinant RT enzymes of both subtype B and C origin were also studied in cell-free reactions.

\section{Results}

Selections with 3TC, FTC, TDF, ABC/3TC and TDF/FTC did not reveal any significant differences between subtype B NL4-3 viruses in terms of mutations acquired. However, exposure of NL4-3 containing subtype $\mathrm{C}$ coding sequences at positions 64/65 to ABC, ATC, d4T, ddI, ABC/ FTC, TDF/3TC and d4T/ddI always resulted in the selection of K65R. This result was not obtained with viruses that contained subtype $C$ coding sequences at either positions 64 or 65 but not both. Most importantly, addition of the silent polymorphisms at TAM sites eased the pressure for the preferential selection of K65R in the newly created NL4-3 (C 64/65/70), NL4-3 (C 64/65/70/219) and NL4-3 (C 64/65/70/210/219) viruses. We have also shown that pausing in cell-free RT reactions occurs preferentially at the 64/65 site and that this is entierly templatedriven and is not dependent on the origin of the RT enzyme employed.

\section{Conclusion}

Silent polymorphisms at both positions 64 and 65 favor the development of $\mathrm{K} 65 \mathrm{R}$ in subtype $\mathrm{C}$ viruses, but this effect can be attenuated by silent polymorphisms at TAM sites 70/210/219. These biochemical and tissue culture findings are of clinical relevance in view of the fact that $\mathrm{d} 4 \mathrm{~T}$-containing regimens are extensively used in developing country settings and because the K65R mutation is also responsible for cross-resistance to multiple members of the N(t)RTI family of drugs. Thus, many second line regimens that might otherwise be considered after failure of a d4T-based regimen might no longer be useful in patients with subtype $\mathrm{C}$ viruses. 